\title{
A Facile and Green Synthesis of Silver Quantum Dots (Ag QDs) Immobilized onto Polymeric CTS-PEO Blend for Photocatalytic Degradation of P-Nitrophenol
}

Fares T. Alshorifi ${ }^{\mathrm{a}, b^{*}}$, Abdullah A. alswat ${ }^{\mathrm{c}}$, Mohammed A. Mannaa ${ }^{\mathrm{d}}$, Mohammed T. Alotaibie, Salah M. El-Bahy ${ }^{e}$, Reda S. Salama ${ }^{\mathrm{f}}$

a Department of chemistry, Faculty of Science, Sheba region University, Yemen

${ }^{\mathrm{b}}$ Department of chemistry, Faculty of Science, Sana'a University, Yemen

${ }^{c}$ Chemistry Department, Faculty of Education and Applied Science -Arhab Sana'a University, Yemen

${ }^{d}$ Chemistry Department, Faculty of Applied Science, Sa'ada University, Yemen.

e Department of Chemistry, Turabah University College, Taif University, P. O. Box 11099, Taif 21944, Saudi Arabia

${ }_{\mathrm{f}}$ Basic Science Department, Faculty of Engineering, Delta University for Science and Technology, Gamasa, Egypt.

*Corresponding author at Department of chemistry, Faculty of Science, Sana'a University, Yemen. E-mail address: amgdfaress@gmail.com (Fares T. Alshorifi). 


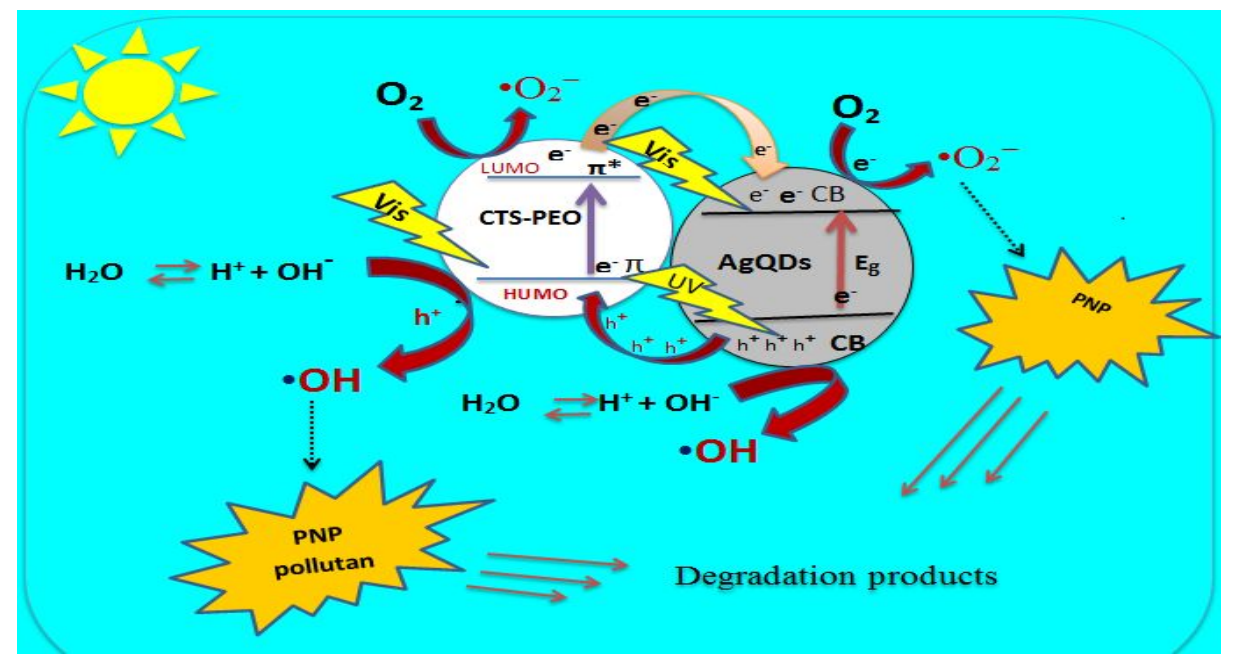

Scheme S1: Proposed mechanism of photocatalytic PNP degradation by Ag QDs/CTS-PEO photocatalysts under a simulated sunlight source. 


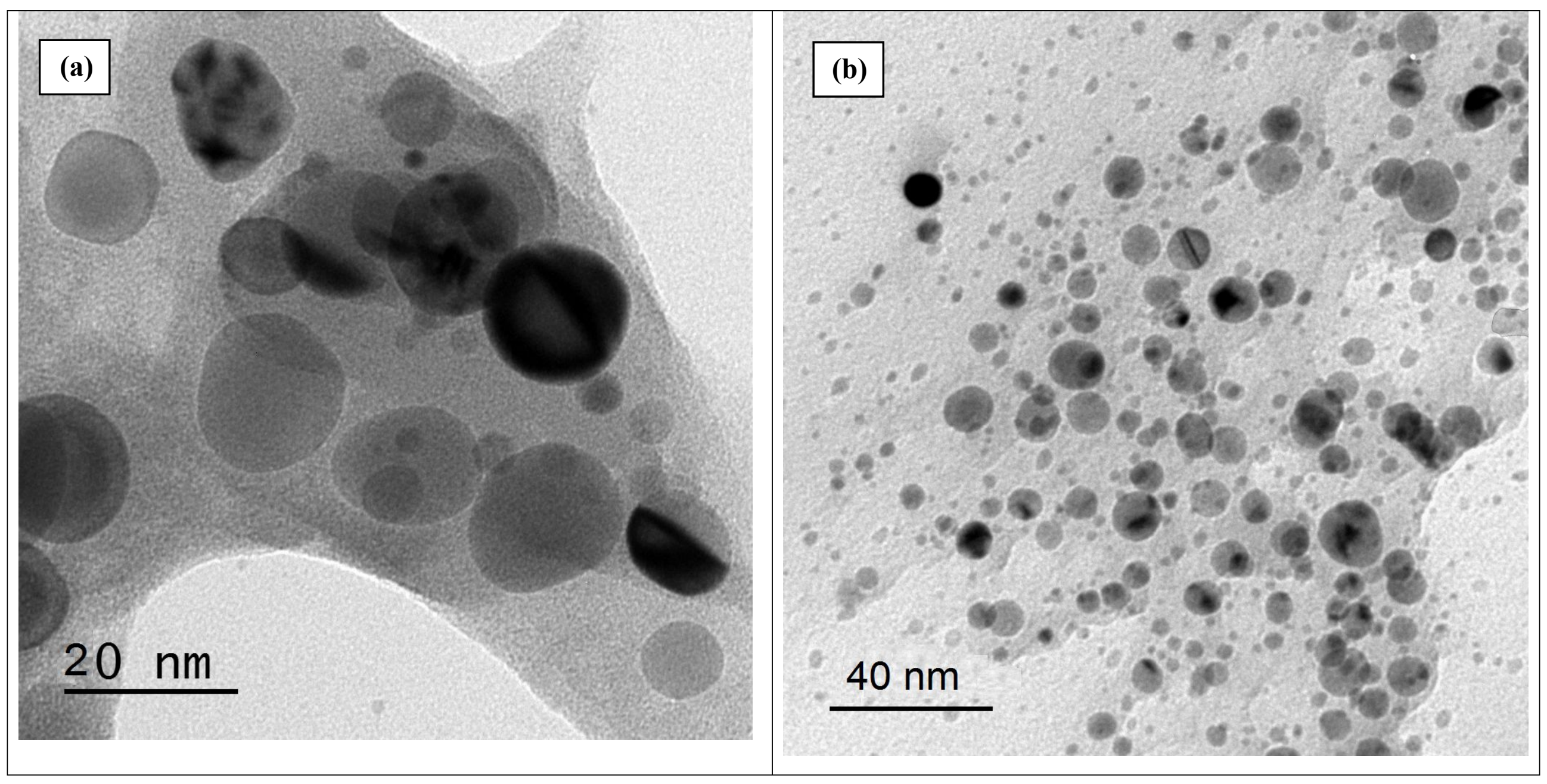




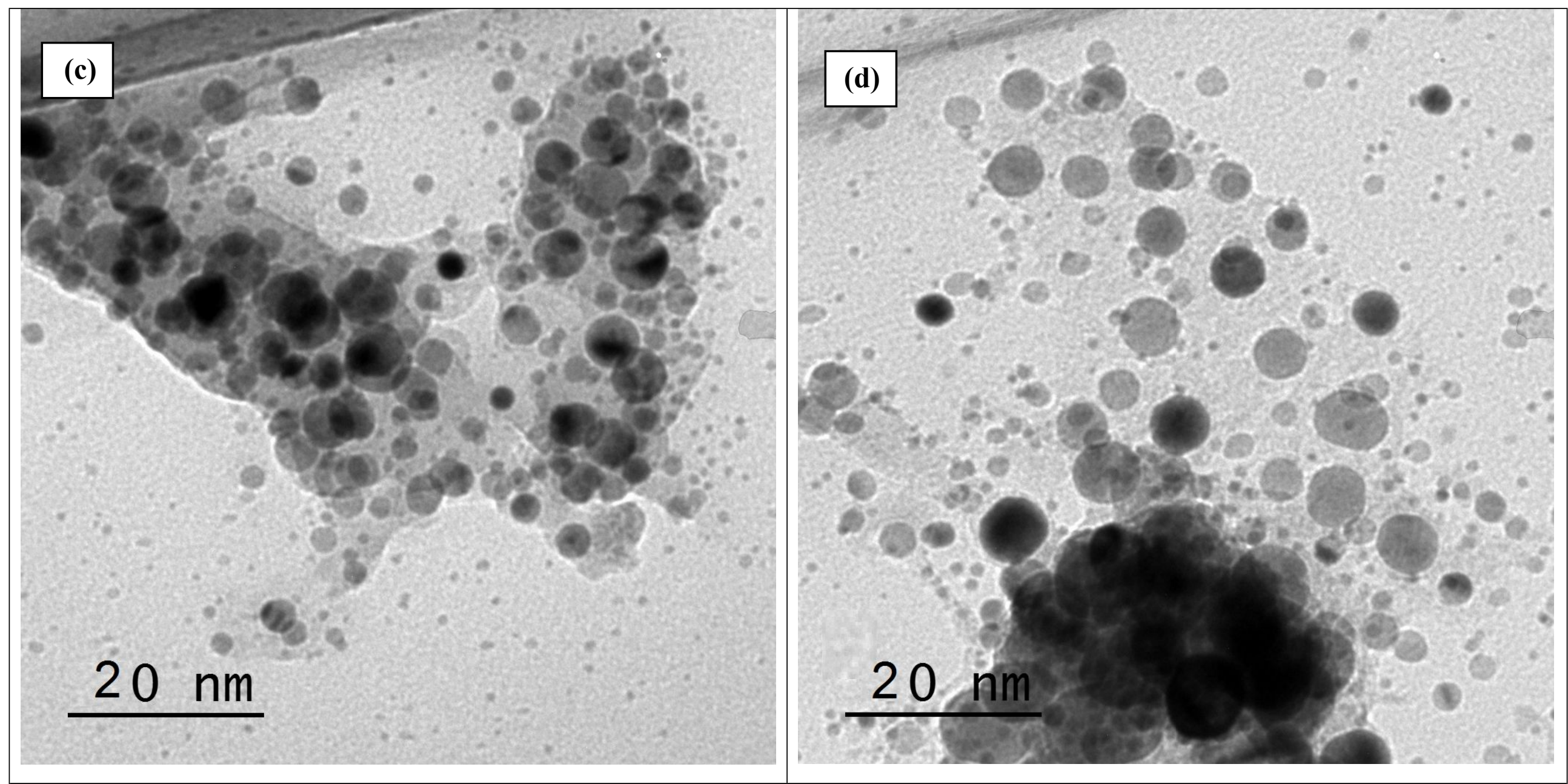

Figure S1: HR-TEM images of (a) 1.0 wt.\%, (b) 6.0 wt.\%, (c) 12.0 wt.\% and (d) 20.0 wt.\% Ag QDs/CTS-PEO. 


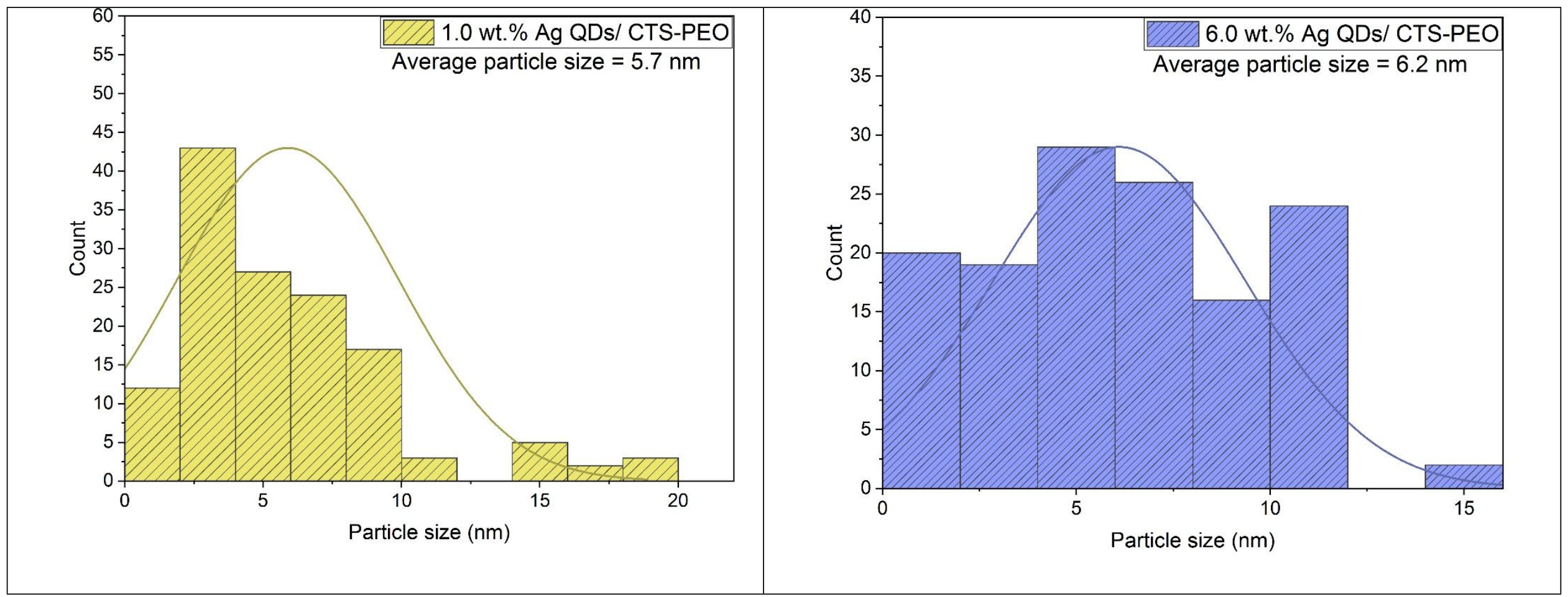




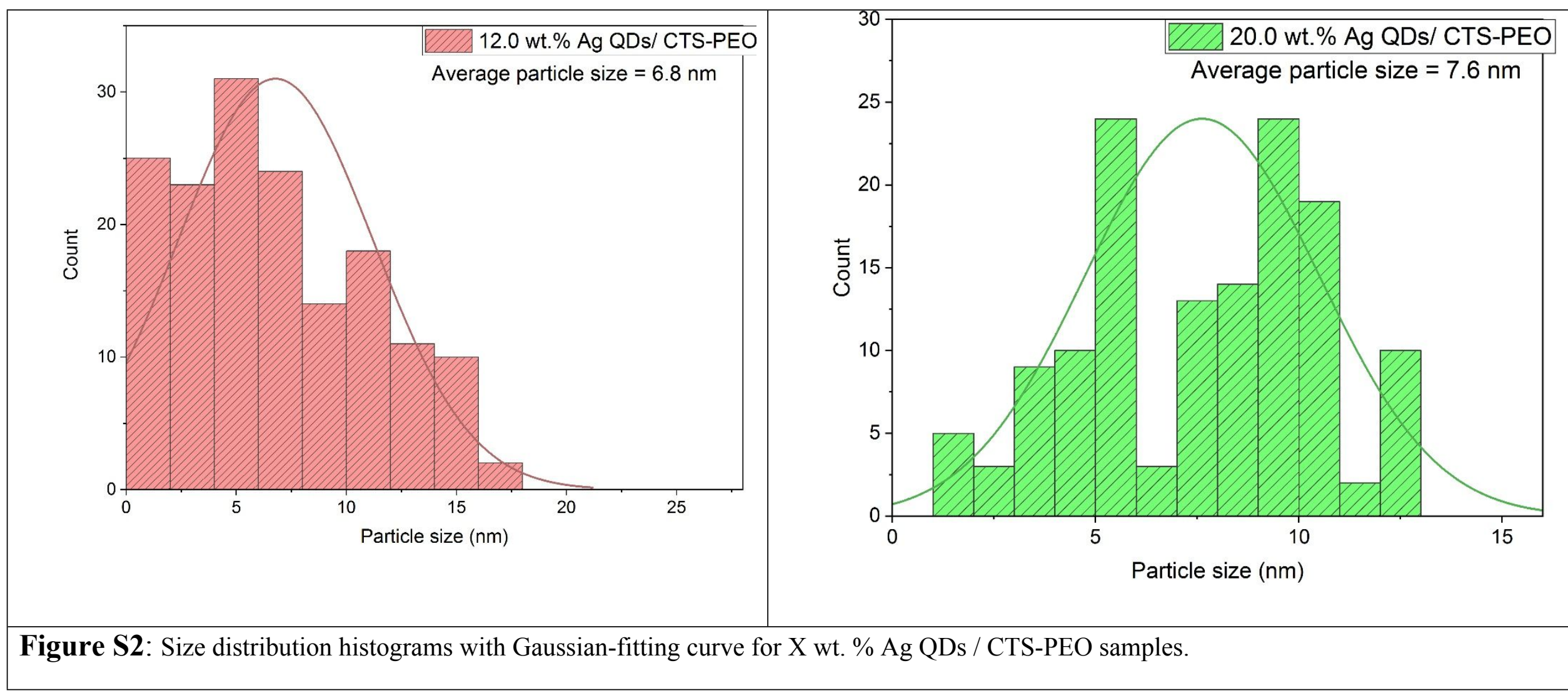




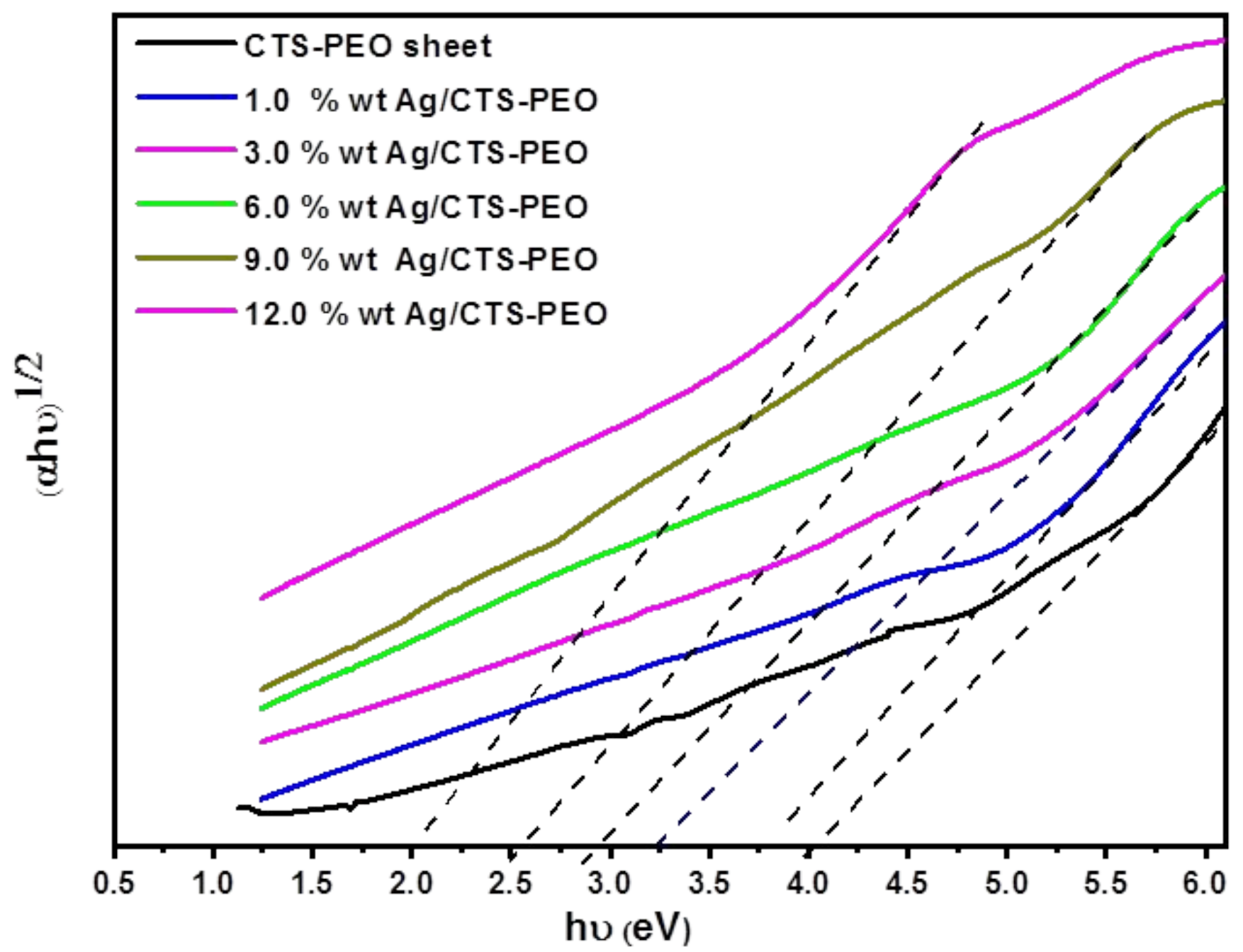

Figure S3: $(\alpha h v)^{1 / 2}$ versus photon energy (hv) for pure CTS-PEO blend and modified Ag QDs/CTS-PEO. 


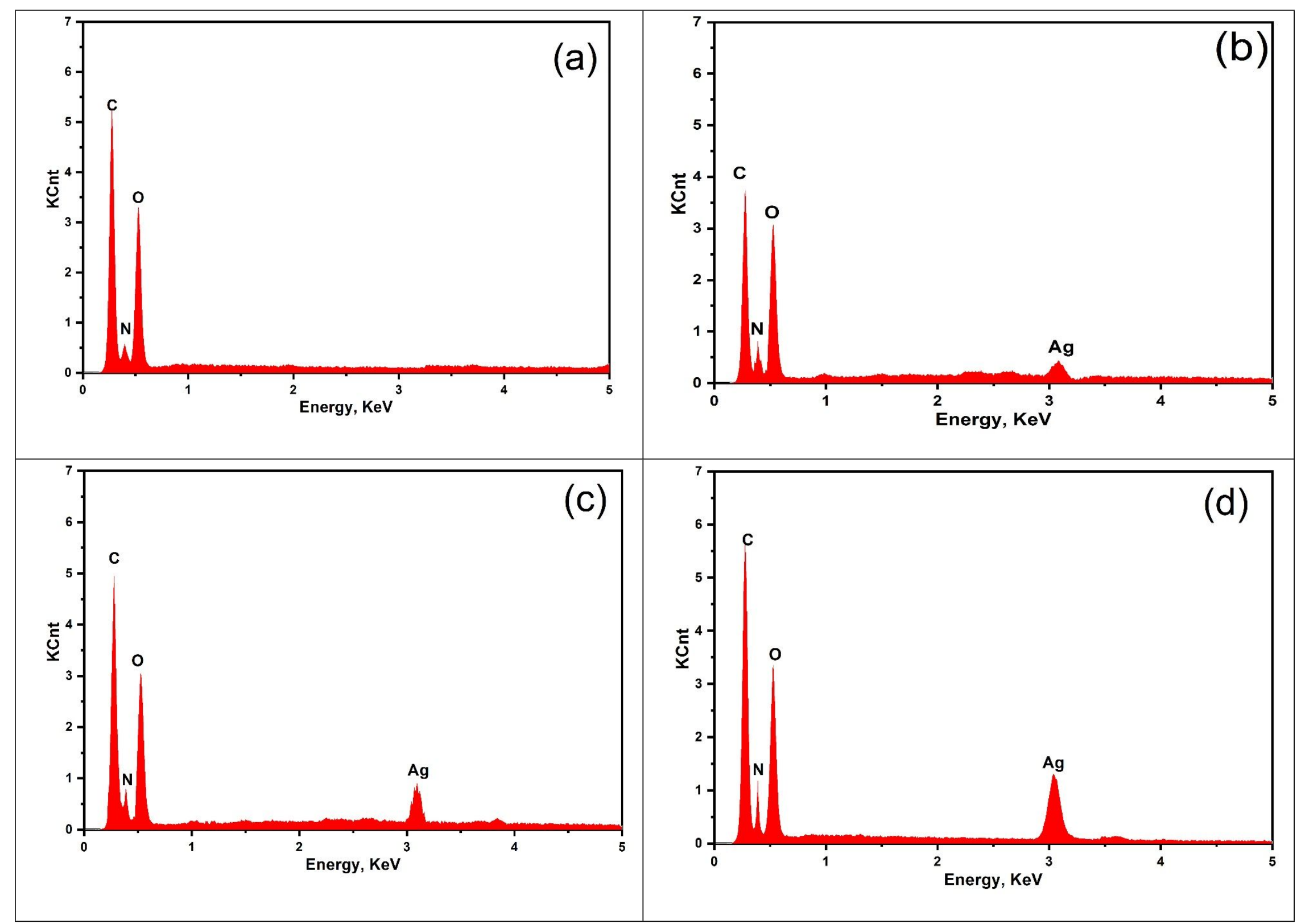




(e)
(c) $6.0 \mathrm{wt.} \%$, (d) $12.0 \mathrm{wt.} \%$ and (e) $20.0 \mathrm{wt.} \% \mathrm{Ag}$
QDs/CTS-PEO..




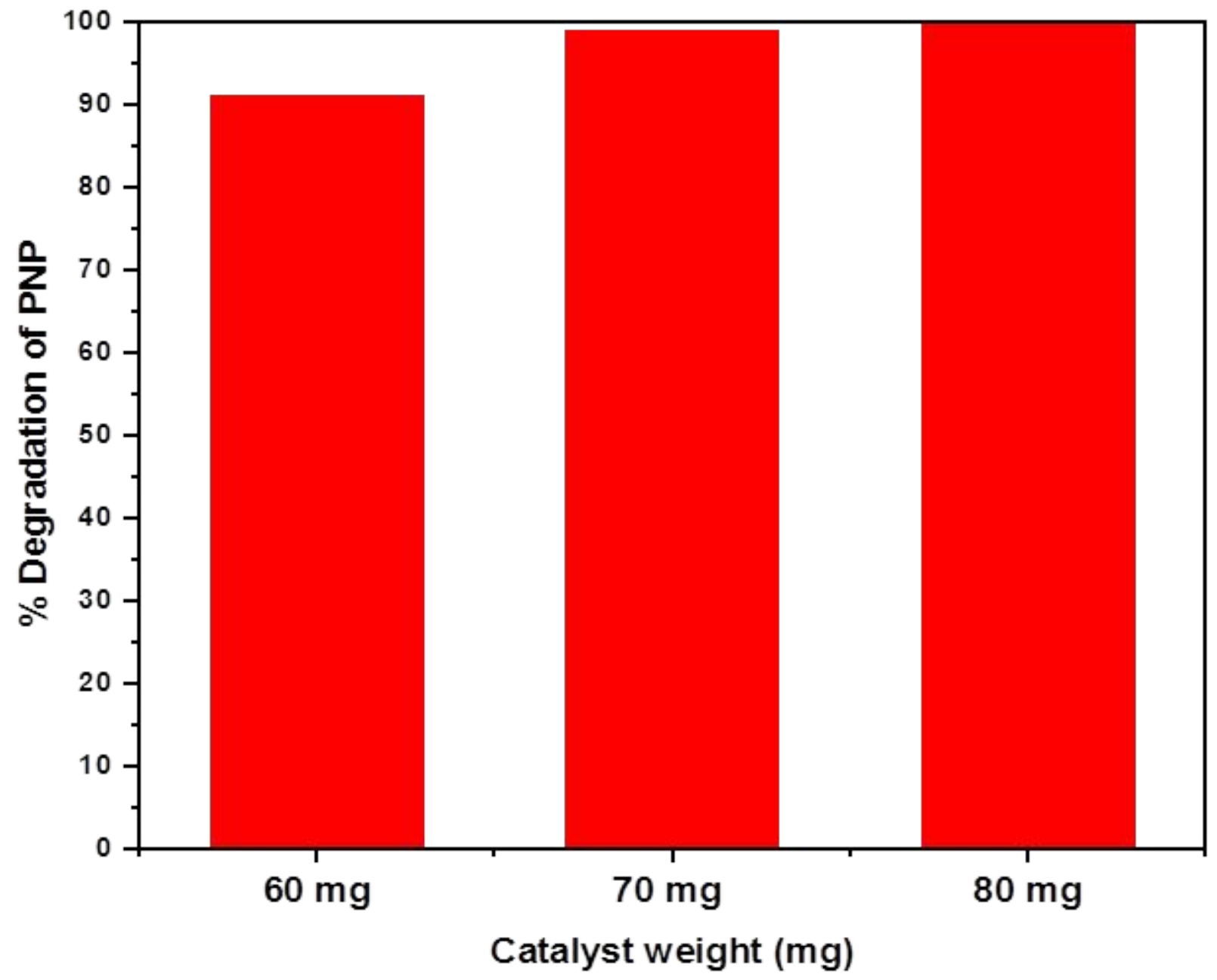

Figure S5: effect of catalyst dosage on the photodegradation of PNP over 12 wt.\% Ag QDs/CTS-PEO. 
Table S1: Comparison of degradation efficiency of Ag QDs/CTS-PEO and other photocatalysts:

\begin{tabular}{ccccc}
\hline No. & photocatalyst & Degradation rate (\%) & Irradiation Time (min) & Reference \\
\hline 1 & Ag QDs/CTS-PEO & $92.1 \%$ & 120 & Current wok \\
2 & $\mathrm{Ag}-\mathrm{TiO}_{2} /$ Porous polymer composite & $81.4 \%$ & 240 & {$[75]$} \\
3 & $\mathrm{Ag} / \mathrm{ZnO} / \mathrm{PMMA}$ Nanocomposites & $90.0 \%$ & 240 & {$[77]$} \\
4 & $\mathrm{PAN}-\mathrm{g}-\mathrm{Alg} @ \mathrm{Ag}$ nanocatalysts & $95.2 \%$ & 120 & {$[78]$} \\
5 & $\mathrm{ZNO}-\mathrm{Ag} / \mathrm{PS}$ & $97.0 \%$ & 120 & {$[79]$} \\
\hline
\end{tabular}

